\title{
Castelnuovo-Mumford Regularity and Hilbert Coefficients of Parameter Ideals
}

\author{
Cao Huy Linh
}

Dedicated to Prof. Ngo Viet Trung on the occasion of his 65-th birthday

\begin{abstract}
Let $A$ be a noetherian local ring of dimension $d \geq 1$ and $\operatorname{depth}(A) \geq d-1$. In this paper, we study the non-positivity for the Hilbert coefficients of parameter ideals in the ring $A$. Moreover, we establish a bound for the Castelnuovo-Mumford regularity of associated graded ring of $A$ with respect to parameter ideal in terms of the first Hilbert coefficient and the dimension.
\end{abstract}

\section{Introduction}

Let $(A, \mathfrak{m})$ be a noetherian local ring of dimension $d$ and $J$ an $\mathfrak{m}$-primary ideal of $A$. Let $\ell(\cdot)$ denote the length of an $A$-module. The Hilbert-Samuel function of $A$ with respect to $J$ is the function $H_{J}: \mathbb{Z} \rightarrow \mathbb{N}_{0}$ given by

$$
H_{J}(n)= \begin{cases}\ell\left(A / J^{n}\right) & \text { if } n \geq 0 \\ 0 & \text { if } n<0\end{cases}
$$

It is well known that there exists a unique polynomial $P_{J}(x) \in \mathbb{Q}[x]$ (called the HilbertSamuel polynomial) of degree $d$ such that $H_{J}(n)=P_{J}(n)$ for $n \gg 0$. We can always write $P_{J}(n)$ of the form

$$
P_{J}(n)=\sum_{i=0}^{d}(-1)^{i}\left(\begin{array}{c}
n+d-i-1 \\
d-i
\end{array}\right) e_{i}(J) .
$$

Then, the integers $e_{i}(J)$ is called Hilbert coefficients of $J$.

If denote by $G(J)=\bigoplus_{n \geq 0} J^{n} / J^{n+1}$ the associated graded ring of $A$ with respect to $J$ and $a_{i}(G(J))=\sup \left\{n \mid H_{G(J)_{+}}^{i}(G(J))_{n} \neq 0\right\}$, then the Castelnuovo-Mumford regularity of $G(J)$ is defined by

$$
\operatorname{reg}(G(J))=\max \left\{a_{i}(G(J))+i \mid i \geq 0\right\}
$$

Received November 3, 2018; Accepted January 21, 2019.

Communicated by Keiichi Watanabe.

2010 Mathematics Subject Classification. Primary: 13D45, 13D07; Secondary: 14B15.

Key words and phrases. Hilbert coefficients, the depth of associated graded rings, parameter ideals, Castelnuovo-Mumford regularity, postulation number.

The author is partially supported by a fund of Vietnam National Foundation for Science and Technology Development (NAFOSTED) under grant number 101.04-2015.32. 
The aim of this paper is to study the non-positivity of the Hilbert coefficients and establish a bound for the Castelnuovo-Mumford regularity of associated graded ring in terms of Hilbert coefficients.

Hilbert coefficients contain structural information of rings and modules, so they have been attracted an attention of many mathematicians. In 2008, Vasconcelos [22 named $e_{1}(J)$ the Chern coefficient. It is well known that the Chern coefficient $e_{1}(Q) \leq 0$ for every parameter ideal $Q$ (see Mandal-Singh-Verma [14]), while other Hilbert coefficients of parameter ideal would be positive. However, if $\operatorname{depth}(A) \geq d-1$, McCune [16] showed that $e_{2}(Q) \leq 0$ for every parameter ideal $Q$. In [20], Saikia and Saloni proved that if $\operatorname{depth}(A) \geq d-1$, then $e_{3}(Q) \leq 0$ for every parameter ideal $Q$. In [16], McCune also proved that if $Q$ is a parameter ideal such that $\operatorname{depth}(G(Q)) \geq d-1$, then $e_{i}(Q) \leq 0$ for $i=1, \ldots, d$. Recently, Saikia-Saloni [20] and Linh-Trung 13 proved that if $\operatorname{depth}(A) \geq$ $d-1$ and $Q$ is a parameter ideal such that $\operatorname{depth}(G(Q)) \geq d-2$, then $e_{i}(Q) \leq 0$ for $i=1, \ldots, d$. This result is an improvement of the Mccune's result in 16 .

In [4], Elias proved that $\operatorname{depth}\left(G\left(Q^{k}\right)\right)$ is constant for $k \gg 0$. We denote this number $\sigma(Q)$. By giving a criterion for the $\sigma(Q) \geq s$, we get the first main result on the nonpositivity of Hilbert coefficients of parameter ideal.

Proposition 3.5. Let $(A, \mathfrak{m})$ be a noetherian local ring of dimension $d \geq 2$ and $\operatorname{depth}(A) \geq$ $d-1$. Let $Q$ be a parameter ideal such that $\sigma(Q) \geq d-2$. Then $e_{d}(Q) \leq 0$.

This implies that if $d=4, \operatorname{depth}(A) \geq 3$ and $\sigma(Q) \geq 2$, then $e_{i}(Q) \leq 0$ for $i=1,2,3,4$. From Proposition 3.5, it follows an early result of Saikia-Saloni [20] and Linh-Trung [13]. As a consequence, it follows that if $d \geq 3$ and $\operatorname{depth}(A) \geq d-1$, then $e_{i}(Q) \leq 0$ for $i=1,2,3$.

Next, we want to establish a bound for the regularity of $G(Q)$ in terms of Hilbert coefficients. It is well known that the regularity of $G(Q)$ provides bounds for some important invariants such as postulation number, relation type, reduction number. There have been many results relate to this issue. In [18], Rossi-Trung-Valla used Hilbert function to establish a bound for the regularity of $G(\mathfrak{m})$ in terms of extended degree and dimension. Later, this result was extended on the class of $\mathfrak{m}$-primary ideals (see [2,10,11]). In [12], Linh-Trung gave a uniform bound for the regularity of associated graded ring with respect to parameter ideal in generalized Cohen-Macaulay ring. Goto-Ozeki [6] used the result of Linh-Trung [12] to establish a uniform bound for Hilbert coefficients of parameter ideal in generalized Cohen-Macaulay ring. They also proved that a ring is generalized Cohen-Macaulay if and only if Hilbert coefficients $e_{i}(Q)$ of parameter ideals are finite, for $i=1, \ldots, d$. It is natural to ask the following question:

Is there any bound for the regularity of associated graded ring $G(Q)$ in terms of Hilbert coefficients $e_{i}(Q)$ for $i=1, \ldots, d$ ? 
In this paper, we establish a bound for the regularity of associated graded $\operatorname{ring} G(Q)$ in terms of the first Hilbert coefficient $e_{1}(Q)$ in the case the ring $A$ satisfies $\operatorname{depth}(A) \geq d-1$.

Theorem 4.5. Let $A$ be a noetherian local ring of dimension $d \geq 1$ and $\operatorname{depth}(A) \geq d-1$. Let $Q$ be a parameter ideal of $A$. Then

$$
\operatorname{reg}(G(Q)) \leq \begin{cases}\max \left\{-e_{1}(Q)-1,0\right\} & \text { if } d=1 \\ \max \left\{\left[-4 e_{1}(Q)\right]^{(d-1) !}+e_{1}(Q)-1,0\right\} & \text { if } d \geq 2\end{cases}
$$

From this we get bounds for the postulation number and relation type of a parameter ideal in terms of the first Hilbert coefficient.

The paper is organized as follows. In Section 2, we prepare some facts relate to Hilbert coefficients and regularity. In Section 3, we prove the non-positivity for Hilbert coefficients of parameter ideals. In Section 4, we establish a bound for the regularity of associated graded ring with respect to parameter ideal in terms of the first Hilbert coefficient.

\section{Preliminary}

Let $R=\bigoplus_{n \geq 0} R_{n}$ be a finitely generated standard graded algebra over a noetherian commutative ring $R_{0}$. Let $R_{+}=\bigoplus_{n>0} R_{n}$. Let $E$ be a finitely generated graded $R$ module with $\operatorname{dim}(E)=d$. Denote by $H_{R_{+}}^{i}(E)$ the $i$-th local cohomological module of $E$ with support ideal $R_{+}$. Define

$$
a_{i}(E):= \begin{cases}\max \left\{n \mid H_{R_{+}}^{i}(E)_{n} \neq 0\right\} & \text { if } H_{R_{+}}^{i}(E) \neq 0 \\ -\infty & \text { if } H_{R_{+}}^{i}(E)=0\end{cases}
$$

The Castelnuovo-Mumford regularity of $E$ is the number

$$
\operatorname{reg}(E):=\max \left\{a_{i}(E)+i \mid i \geq 0\right\}
$$

If the basic ring $R_{0}$ of $R$ is artinian, $h_{E}(n):=\ell\left(E_{n}\right)$ denote the Hilbert function of $E$. The unique polynomial $p_{E}(X)$ for which $h_{E}(n)=p_{E}(n)$ for $n \gg 0$ is called the Hilbert polynomial of $E$. It is written in the form

$$
p_{E}(n)=\sum_{i=0}^{d-1}(-1)^{i}\left(\begin{array}{c}
n+d-i-1 \\
d-i-1
\end{array}\right) e_{i}(E)
$$

where $e_{i}(E)$ for $i=0,1, \ldots, d-1$ are integers, called the Hilbert coefficients of $E$. The postulation number $p(E)$ of $E$ is defined to be the integer number

$$
p(E)=\max \left\{n \mid h_{E}(n) \neq p_{E}(n)\right\} .
$$


A relationship between Hilbert function and Hilbert polynomial is given by the following formula (see [15, Lemma 1.3]):

$$
h_{E}(n)-p_{E}(n)=\sum_{i=0}^{d}(-1)^{i} \ell\left(H_{R_{+}}^{i}(E)_{n}\right) .
$$

From this formula, we get the following inequality.

Lemma 2.1. With assumption as above, we have

$$
p(E) \leq \max \left\{a_{0}(E), \ldots, a_{d}(E)\right\} \leq \operatorname{reg}(E) .
$$

Now, let $(A, \mathfrak{m})$ be a noetherian local ring of dimension $d$ and $J$ be an $\mathfrak{m}$-primary ideal of $A$. A numerical function

$$
H_{J}: \mathbb{Z} \longrightarrow \mathbb{N}_{0}, \quad n \longmapsto H_{J}(n)= \begin{cases}\ell\left(A / J^{n}\right) & \text { if } n \geq 0, \\ 0 & \text { if } n<0\end{cases}
$$

is said to be a Hilbert-Samuel function of $A$ with respect to the ideal $J$. It is well known that there exists a polynomial $P_{J} \in \mathbb{Q}[x]$ of degree $d$ such that $H_{J}(n)=P_{J}(n)$ for $n \gg 0$. The polynomial $P_{J}$ is called the Hilbert-Samuel polynomial of $A$ with respect to the ideal $J$ and it is written of the form

$$
P_{J}(n)=\sum_{i=0}^{d}(-1)^{i}\left(\begin{array}{c}
n+d-i-1 \\
d-i
\end{array}\right) e_{i}(J),
$$

where $e_{i}(J)$ for $i=0, \ldots, d$ are integers, called Hilbert coefficients of $J$. In particular, $e(J)=e_{0}(J)$ and $e_{1}(J)$ are called the multiplicity and Chern coefficient of $J$, respectively. Denote

$$
n(J)=\max \left\{n \mid H_{J}(n) \neq P_{J}(n)\right\} .
$$

Let $G(J)=\bigoplus_{n \geq 0} J^{n} / J^{n+1}$ denote the associated graded module of $A$ with respect to $J$. Then,

$$
e_{i}(G(J))=e_{i}(J) \quad \text { for } i=0, \ldots, d-1 .
$$

Lemma 2.2. 2, Lemma 3.5]

$$
n(J)=p(G(J)) .
$$

Let $d=\operatorname{dim}(A) \geq 1, L=H_{\mathfrak{m}}^{0}(A), \bar{A}=A / L$ and $\bar{J}=J \bar{A}$. We have

$$
\begin{aligned}
\ell\left(A / J^{n}\right) & =\ell\left(A /\left(J^{n}+L\right)+\ell\left(J^{n}+L / I^{n}\right)\right. \\
& =\ell\left(\bar{A} / \bar{J}^{n}\right)+\ell\left(L / J^{n} \cap L\right) .
\end{aligned}
$$

Since $\ell(L)<\infty, I^{n} \cap L=0$ for $n \gg 0$. Thus,

$$
\ell\left(A / J^{n}\right)=\ell\left(\bar{A} / \bar{J}^{n}\right)+\ell(L) \quad \text { for } n \gg 0 .
$$

This allows us to state the following lemma. 
Lemma 2.3. If $d=\operatorname{dim}(A) \geq 1$ then

(i) $e_{i}(J)=e_{i}(\bar{J})$ for $i=0, \ldots, d-1$;

(ii) $e_{d}(J)=e_{d}(\bar{J})+(-1)^{d} \ell(L)$.

If $d=1$ and $I=Q$ is a parameter ideal of $A$, then $\bar{A}$ is Cohen-Macaulay. This implies that $e_{1}(\bar{Q})=0$. From this we get the following corollary.

Corollary 2.4. If $\operatorname{dim}(A)=1$ and $Q$ is a parameter ideal of $A$, then

$$
e_{1}(Q)=-\ell(L)
$$

An element $x \in J \backslash \mathfrak{m} J$ is said to be superficial for $J$ if there exists a number $c \in \mathbb{N}$ such that $\left(J^{n}: x\right) \cap J^{c}=J^{n-1}$ for $n>c$. If $A / \mathfrak{m}$ is infinite, then a superficial element for $J$ always exists. A sequence of elements $x_{1}, \ldots, x_{r} \in J \backslash \mathfrak{m} J$ is said to be a superficial sequence for $J$ if $x_{i}$ is superficial for $J /\left(x_{1}, \ldots, x_{i-1}\right)$ for $i=1, \ldots, r$. Suppose that $\operatorname{dim}(A)=d \geq 1$ and $x \in J \backslash \mathfrak{m} J$ is a superficial element for $J$, then $\ell\left(0:_{A} x\right)<\infty$ and $\operatorname{dim}(A /(x))=\operatorname{dim}(A)-1=d-1$. The following lemma give us a relationship between $e_{i}(J)$ and $e_{i}(\bar{J})$, where $\bar{J}=J(A /(x))$.

Lemma 2.5. [19, Proposition 1.3.2] Let $A$ be a noetherian local ring of dimension $d \geq 2$ and $J$ be an $\mathfrak{m}$-primary ideal of $A$. Let $x \in J \backslash \mathfrak{m} J$ be a superficial element for $J$ and $\bar{J}=J(A /(x))$. Then

(i) $e_{i}(J)=e_{i}(\bar{J})$ for $i=0, \ldots, d-2$;

(ii) $e_{d-1}(J)=e_{d-1}(\bar{J})+(-1)^{d} \ell(0: x)$.

3. The non-positivity of Hilbert coefficients of parameter ideals

Through this section, let $(A, \mathfrak{m})$ be a noetherian local ring of dimension $d, I$ be an $\mathfrak{m}$ primary ideal of $A$ and $y_{1}, \ldots, y_{d} \in I$. For $i=1, \ldots, d-1$, let $A_{i}=A /\left(y_{1}, \ldots, y_{i}\right)$ and $I_{i}=I A_{i}$. Denote by $G\left(I_{k}\right)$ the associated graded ring of $A_{k}$ with respect to $I_{k}$.

Lemma 3.1. 9, Lemma 2.2] Let $(A, \mathfrak{m})$ be a noetherian local ring and $I$ be an $\mathfrak{m}$-primary ideal of $A$. For $1 \leq k \leq d-1$, let $y_{1}, \ldots, y_{k}$ be a superficial sequence for $I$. Then,

$$
\operatorname{depth}\left(G\left(I_{k}\right)\right) \geq 1 \quad \text { if and only if } \operatorname{depth}(G(I)) \geq k+1
$$

Let $J$ be an ideal of $A$. In [4, Proposition 2.2], Elias proved that $\operatorname{depth}\left(G\left(J^{k}\right)\right)$ is constant for $k \gg 0$. We call this number $\sigma(J)$. The following lemma gives whenever the number $\sigma(J)$ is positive. 
Lemma 3.2. Let $J$ be an $\mathfrak{m}$-primary ideal of $A$. If $\operatorname{depth}(A) \geq 1$ then $\sigma(J) \geq 1$.

Proof. Without loss of generality, we may assume that the residue field $A / \mathfrak{m}$ is infinite. Suppose that $x$ is a superficial element for $J$. Then $y=x^{k}$ is also a superficial element for $I=J^{k}$. Since $\operatorname{depth}(A) \geq 1, J^{k}: x=J^{k-1}$ for $k \gg 0$. To prove $\sigma(J)>0$, we show that $\operatorname{depth}\left(G\left(J^{k}\right)\right)=\operatorname{depth}(G(I))>0$ for $k \gg 0$. Indeed, for all $k \gg 0$ and $n \geq 1$ we have

$$
\begin{aligned}
I^{n}: y & =J^{n k}: x^{k}=\left(J^{n k}: x\right): x^{k-1}=J^{n k-1}: x^{k-1} \\
& =\cdots=J^{n k-k}=J^{k(n-1)}=I^{n-1} .
\end{aligned}
$$

Therefore $\operatorname{depth}(G(I))>0$. This implies that $\sigma(J)>0$.

However, the $\operatorname{depth}(A) \geq s$ does not imply $\sigma(J) \geq s$ for $s \geq 2$. The following proposition gives a criterion for $\sigma(J) \geq s$.

Proposition 3.3. Let $(A, \mathfrak{m})$ be a noetherian local ring of dimension $d \geq 3$ and $\operatorname{depth}(A) \geq$ $r$. Let $J$ be an $\mathfrak{m}$-primary ideal and $x_{1}, \ldots, x_{s}$ be a superficial sequence for $J, 2 \leq s \leq r$. For $k \gg 0$, let $I=J^{k}$ and $y_{1}=x_{1}^{k}, \ldots, y_{s}=x_{s}^{k}$. Set $A_{i}=A /\left(y_{1}, \ldots, y_{i}\right)$ and $I_{i}=I A_{i}$. The following conditions are equivalent.

(i) $\sigma(J) \geq s$;

(ii) $\left(I_{s-1}^{n}: y_{s} A_{s-1}\right)=I_{s-1}^{n-1}$ for all $n=2, \ldots, s$;

(iii) $I^{n} \cap\left(y_{1}, \ldots, y_{s}\right)=\left(y_{1}, \ldots, y_{s}\right) I^{n-1}$ for all $n=2, \ldots, s$.

Proof. (i) $\Rightarrow$ (ii). Denote $G\left(I_{s-1}\right)$ the associated graded ring of $A_{s-1}$ with respect to $I_{s-1}$. By Lemma 3.1, $\operatorname{depth}(G(I)) \geq s$ if and only if $\operatorname{depth} G\left(I_{s-1}\right) \geq 1$. This is equivalent to $\left(I_{s-1}^{n+1}: y_{s} A_{s-1}\right)=I_{s-1}^{n}$ for all $n \geq 1$; so, the implication from (i) to (ii) is evident.

(ii) $\Rightarrow$ (i). By [7, Lemma 2.4], $a_{i}(G(I)) \leq 0$ for $k \gg 0$ and for all $i \geq 0$. Let $y_{1}^{*}$ denote the initial form of $y_{1}$ in $G(I)$. From [21, Lemma 2.3], we have

$$
a_{i+1}(G(I))+1 \leq a_{i}\left(G(I) / y_{1}^{*} G(I)\right) \leq \max \left\{a_{i}(G(I)), a_{i+1}(G(I))+1\right\} .
$$

But

$$
H_{G(I)_{+}}^{i}\left(G(I) /\left(y_{1}^{*} G(I)\right)\right) \cong H_{G\left(I_{1}\right)_{+}}^{i}\left(G\left(I_{1}\right)\right) \text { for all } i=1, \ldots, d-1
$$

and $a_{0}\left(G\left(I_{1}\right)\right) \leq a_{0}\left(G(I) /\left(y_{1}^{*}\right)\right)$. Therefore $a_{i}\left(G\left(I_{1}\right)\right) \leq 1$ for $i \geq 0$. Similarly argument, we get $a_{i}\left(G\left(I_{j}\right)\right) \leq j$ for all $i \geq 0$ and $j=1, \ldots, s$. By Lemmas 2.1 and $2.2, n\left(I_{j}\right) \leq j$ for $j=1, \ldots, s$. From an exact sequence

$$
0 \rightarrow\left(I_{s-1}^{n+1}: y_{s} A_{s-1}\right) / I_{s-1}^{n} \rightarrow A_{s-1} / I_{s-1}^{n} \stackrel{y_{s}}{\rightarrow} A_{s-1} / I_{s-1}^{n+1} \rightarrow A_{s} /\left(I_{s}^{n+1}\right) \rightarrow 0
$$


we get

$$
\begin{aligned}
\ell\left(I_{s-1}^{n+1}: y_{s} A_{s-1} / I_{s-1}^{n}\right) & =\ell\left(A_{s} /\left(I_{s}^{n+1}\right)\right)-\ell\left(A_{s-1} / I_{s-1}^{n+1}\right)+\ell\left(A_{s-1} / I^{n} A_{s-1}\right) \\
& =\ell\left(A_{s} / I_{s}^{n+1} A_{s}\right)-\ell\left(I^{n} A_{s-1} / I^{n+1} A_{s-1}\right) .
\end{aligned}
$$

Since $n\left(I_{s-1}\right) \leq s-1, n\left(I_{s}\right) \leq s$ and $e_{i}\left(I_{s}\right)=e_{i}\left(I_{s-1}\right)$ for $i=0,1, \ldots, d-s$,

$$
\ell\left(I^{n+1} A_{s-1}: y_{s} A_{s-1} / I^{n} A_{s-1}\right)=0 \text { for all } n \geq s .
$$

Therefore

$$
I^{n+1} A_{s-1}: y_{s} A_{s-1}=I^{n} A_{s-1} \quad \text { for all } n \geq s .
$$

From the hypothesis and (3.1), it follows that $I^{n+1} A_{s-1}: y_{s} A_{s-1}=I^{n} A_{s-1}$ for all $n \geq 1$. Thus $\operatorname{depth}\left(G\left(I_{s-1}\right)\right) \geq 1$. Applying Lemma 3.1. we get $\operatorname{depth}(G(I)) \geq s$.

(ii) $\Rightarrow$ (iii). Suppose that

$$
\left(I_{s-1}^{n}: y_{s} A_{s-1}\right)=I_{s-1}^{n-1} \quad \text { for all } n=2, \ldots, s .
$$

Consider the case $s=1$. From $I^{n}: y_{1}=I^{n-1}$, we have $y_{1}\left(I^{n}: y_{1}\right)=y_{1} I^{n-1}$. This implies $I^{n} \cap\left(y_{1}\right)=y_{1} I^{n-1}$. So, the implication holds for $s=1$.

By induction on $s$, we may assume that

$$
I^{n} \cap\left(y_{1}, \ldots, y_{s-1}\right)=\left(y_{1}, \ldots, y_{s-1}\right) I^{n-1} .
$$

From $\left(I_{s-1}^{n}: y_{s} A_{s-1}\right)=I_{s-1}^{n-1}$ and $y_{s}$ forms $A_{s-1}$-regular, we get

$$
I_{s-1}^{n} \cap\left(y_{s} A_{s-1}\right)=y_{s} I_{s-1}^{n-1} .
$$

Thus

$$
\left[I^{n}+\left(y_{1}, \ldots, y_{s-1}\right)\right] \cap\left(y_{1}, \ldots, y_{s}\right)=y_{s} I^{n-1}+\left(y_{1}, \ldots, y_{s-1}\right)
$$

Therefore

$$
\left[\left(I^{n}+\left(y_{1}, \ldots, y_{s-1}\right)\right) \cap\left(y_{1}, \ldots, y_{s}\right)\right] \cap I^{n}=y_{s} I^{n-1}+\left(y_{1}, \ldots, y_{s-1}\right) \cap I^{n}
$$

It follows that

$$
I^{n} \cap\left(y_{1}, \ldots, y_{s}\right)=y_{s} I^{n-1}+\left(y_{1}, \ldots, y_{s-1}\right) \cap I^{n}
$$

From inductive assumption, $\left(y_{1}, \ldots, y_{s-1}\right) \cap I^{n}=\left(y_{1}, \ldots, y_{s-1}\right) I^{n-1}$. So, we obtain

$$
I^{n} \cap\left(y_{1}, \ldots, y_{s}\right)=\left(y_{1}, \ldots, y_{s}\right) I^{n-1} .
$$

(iii) $\Rightarrow$ (ii). Suppose that

$$
I^{n} \cap\left(y_{1}, \ldots, y_{s}\right)=\left(y_{1}, \ldots, y_{s}\right) I^{n-1} .
$$


It follows that

$$
\left[I^{n}+\left(y_{1}, \ldots, y_{s-1}\right)\right] \cap\left(y_{1}, \ldots, y_{s}\right)=\left(y_{1}, \ldots, y_{s}\right) I^{n-1}+\left(y_{1}, \ldots, y_{s-1}\right)
$$

From this we can write

$$
I_{s-1}^{n} \cap\left(y_{s} A_{s-1}\right)=y_{s} I_{s-1}^{n-1} .
$$

Hence

$$
y_{s} A_{s-1}\left[I_{s-1}^{n}:\left(y_{s} A_{s-1}\right)\right]=y_{s} I_{s-1}^{n-1} .
$$

Since $\operatorname{depth}\left(A_{s-1}\right) \geq 1$,

$$
I_{s-1}^{n}:\left(y_{s} A_{s-1}\right)=I_{s-1}^{n-1} .
$$

In [4, Theorem 2.4], Elias proved (i) is equivalent to (iii).

Proposition 3.4. Let $(A, \mathfrak{m})$ be a noetherian local ring of dimension $d \geq 1$ and $Q$ be a parameter ideal of $A$. Then $e_{1}(Q) \leq 0$.

Proof. See 14 .

Proposition 3.5. Let $(A, \mathfrak{m})$ be a noetherian local ring of dimension $d \geq 2$ and $\operatorname{depth}(A) \geq$ $d-1$. Let $Q$ be a parameter ideal such that $\sigma(Q) \geq d-2$. Then $e_{d}(Q) \leq 0$.

Proof. Let $I=Q^{k}$ for $k \gg 0$. By [5, Proposition 2.8], we have $e_{d}(Q)=e_{d}(I)$. We have $\sigma(Q)=\operatorname{depth}(G(I)) \geq d-2$. By [7, Lemma 2.4], $a_{i}(G(I)) \leq 0$ for all $i \geq 0$. Since $Q$ is a parameter ideal, $a_{d}(G(I)) \leq-1$. On the other hand, by [8, Theorem 5.2], $a_{d-2}(G(I))<a_{d-1}(G(I)) \leq 0$. Let $R=A[I t]=\bigoplus_{n \geq 0} I^{n}$ denote the Rees algebra of $A$ with respect to $I$ and $R_{+}=\bigoplus_{n>0} R_{n}$. By [1, Theorem 4.1] and [1, Theorem 3.8], we have

$$
\begin{aligned}
(-1)^{d} e_{d}(Q) & =(-1)^{d} e_{d}(I)=P_{I}(0)-H_{I}(0)=\sum_{i=0}^{d}(-1)^{i} \ell\left(H_{R_{+}}^{i}(R)_{0}\right) \\
& =\sum_{i=0}^{d}(-1)^{i} \ell\left(H_{G(I)_{+}}^{i} G(I)_{0}\right)=(-1)^{d-1} \ell\left(H_{G(I)_{+}}^{d-1} G(I)_{0}\right) .
\end{aligned}
$$

This implies that $e_{d}(Q)=-\ell\left(H_{G(I)_{+}}^{d-1}(G(I))_{0}\right) \leq 0$.

Corollary 3.6. Let $(A, \mathfrak{m})$ be a noetherian ring of dimension $d=4$ and $\operatorname{depth}(A) \geq 3$. Let $Q$ be a parameter ideal of $A$ such that $\sigma(Q) \geq 2$. Then

$$
e_{i}(Q) \leq 0 \quad \text { for all } i=1,2,3,4 .
$$


Proof. First, from Proposition 3.4, we have $e_{1}(Q) \leq 0$. Applying Proposition 3.5, we get $e_{4}(Q) \leq 0$.

Now, we need to show $e_{2}(Q) \leq 0$ and $e_{3}(Q) \leq 0$. Suppose that $Q=\left(x_{1}, x_{2}, x_{3}, x_{4}\right)$. Without loss of generality, assume that $A / \mathfrak{m}$ is infinite and $x_{1}, x_{2}$ is a superficial sequence for $Q$. For each $i=1,2$, let $A_{1}=A /\left(x_{1}\right), A_{2}=A /\left(x_{1}, x_{2}\right)$ and $Q_{i}=Q A_{i}$. Then $\operatorname{dim}\left(A_{i}\right)=4-i$ and $Q_{i}$ is a parameter ideal of $A_{i}$. $\operatorname{Since} \operatorname{depth}(A) \geq 3, \operatorname{depth}\left(A_{i}\right) \geq 4-i-1$. By Lemma 3.2, $\sigma\left(Q_{i}\right) \geq 1$. By applying Proposition 3.5, we obtain

$$
e_{4-i}(Q)=e_{4-i}\left(Q_{i}\right) \leq 0 \quad \text { for } i=1,2 .
$$

So, $e_{2}(Q) \leq 0$ and $e_{3}(Q) \leq 0$.

If $A$ is a noetherian ring of dimension $d \geq 3$ and $\operatorname{depth}(A) \geq d-1$, by analogously arguing as the proof of Corollary 3.6 we obtain the following corollary.

Corollary 3.7. Let $(A, \mathfrak{m})$ be a noetherian local ring of dimension $d \geq 3$ and $\operatorname{depth}(A) \geq$ $d-1$. Let $Q$ be a parameter ideal of $A$. Then

$$
e_{i}(Q) \leq 0 \quad \text { for } i=1,2,3 .
$$

By [4, Proposition 2.2], $\sigma(Q)=\operatorname{depth} G\left(Q^{k}\right) \geq \operatorname{depth} G(Q)$ for $k \gg 0$. From Theorem 3.6 and [4, Proposition 2.2], we get the following corollary.

Corollary 3.8. Let $(A, \mathfrak{m})$ be a noetherian local ring of dimension $d \geq 2$ and $\operatorname{depth}(A) \geq$ $d-1$. Let $Q$ be a parameter ideal of $A$ such that $\operatorname{depth}(G(Q)) \geq d-2$. Then

$$
e_{i}(Q) \leq 0 \quad \text { for } i=1, \ldots, d
$$

Proof. It is well known that $e_{1}(Q) \leq 0$ and $e_{d}(Q) \leq 0$. If $d \leq 4$, the corollary is proved by Corollary 3.6. If $d>4$, we need to prove that $e_{d-i}(Q) \leq 0$ for $i=1, \ldots, d-2$. Indeed, let $Q=\left(x_{1}, \ldots, x_{d}\right)$. Without loss of generality, assume that $A / \mathfrak{m}$ is infinite and $x_{1}, \ldots, x_{d}$ is a superficial sequence for $Q$. For each $i=1, \ldots, d-2$, let $A_{i}=A /\left(x_{1}, \ldots, x_{i}\right)$ and $Q_{i}=Q A_{i}$. Then $\operatorname{dim}\left(A_{i}\right)=d-i$ and $Q_{i}$ is a parameter ideal of $A_{i}$. From $\operatorname{depth}(A) \geq d-1$, $\operatorname{depth}\left(A_{i}\right) \geq d-i-1$. Since $\operatorname{depth}(G(Q)) \geq d-2, \operatorname{depth}(G(Q)) \geq d-i-2$. Вy 4 , Proposition 2.2], $\sigma\left(Q_{i}\right) \geq \operatorname{depth}\left(G\left(Q_{i}\right)\right) \geq d-i-2$. By applying Proposition 3.5, we get

$$
e_{d-i}(Q)=e_{d-i}\left(Q_{i}\right) \leq 0 \quad \text { for } i=1, \ldots, d-2 .
$$

Hence $e_{i}(Q) \leq 0$ for $i=2, \ldots, d-1$.

Notice that Corollary 3.8 is an early results of Saikia-Saloni 20, Corollary 3.2] and Linh-Trung [13, Theorem 2.9].

The assumption $\operatorname{depth}(A) \geq d-1$ is very important. The following example show that some Hilbert coefficients of parameter ideal would be positive if the $\operatorname{ring} A$ is not almost Cohen-Macaulay. 
Example 3.9. [16, Example 3.7] Let $A=k[x, y, z, u, v, w] / I$, where $I=(x+y, z-$ $u, w) \cap(z, u-v, y) \cap(x, u, w)$ and $Q=(u-y, z+w, x-v)$. Then $A$ is an unmixed ring of dimension $d=3, \operatorname{depth}(A)=1$ and $Q$ is a parameter ideal with

$$
P_{Q}(n)=3\left(\begin{array}{c}
n+2 \\
3
\end{array}\right)+2\left(\begin{array}{c}
n+1 \\
2
\end{array}\right)+n \text {. }
$$

In particular, $e_{2}(Q)=1>0$.

4. Bound for the regularity in terms of the first Hilbert coefficients

Let $(A, \mathfrak{m})$ be a noetherian local ring of dimension $d$. Let $Q$ be a parameter ideal of $A$. In this section, we will establish a bound for the regularity of $G(Q)$ in terms of $e_{1}(Q)$ and $d$ in the case $\operatorname{depth}(A) \geq d-1$. First, we need to give a bound for Hilbert-Samuel function of $A$ with respect to $Q$.

Lemma 4.1. Let $A$ be a noetherian local ring of dimension $d \geq 1$ and $\operatorname{depth}(A) \geq d-1$. Let $Q$ be a parameter ideal of $A$. For all $n \geq 1$,

$$
\ell\left(A / Q^{n}\right) \leq\left(\begin{array}{c}
n+d-1 \\
d
\end{array}\right) e(Q)-\left(\begin{array}{c}
n+d-2 \\
d-1
\end{array}\right) e_{1}(Q) .
$$

Proof. Let $L$ denote the largest ideal of finite length of $A$. From the equality

$$
\ell\left(A / Q^{n}\right)=\ell\left(A /\left(Q^{n}+L\right)\right)+\ell\left(Q^{n}+L / Q^{n}\right),
$$

we get

$$
\ell\left(A / Q^{n+1}\right) \leq \ell\left(A / Q^{n+1}+L\right)+\ell(L)
$$

If $d=1$, then $A / L$ is an one-dimensional Cohen-Macaulay ring and $L=H_{\mathfrak{m}}^{0}(A)$. By Corollary 2.4. $e_{1}(Q)=-\ell(L)$. Therefore

$$
\ell\left(A / Q^{n+1}+L\right)+\ell(L)=(n+1) e(Q)-e_{1}(Q),
$$

which proves this case.

If $d>1$, let $Q=\left(x_{1}, \ldots, x_{d}\right)$ and assume that $x=x_{1}$ is superficial for $Q$. Let $\bar{A}=A /(x), \bar{Q}=Q \bar{A}$. Then $e(\bar{Q})=e(Q)$ and $e_{1}(\bar{Q})=e_{1}(Q)$ by Lemma 2.5. Using induction on $d$ we may assume that for all $i \geq 1$,

$$
\begin{aligned}
\ell\left(\bar{A} / \bar{Q}^{i}\right) & \leq\left(\begin{array}{c}
i+d-2 \\
d-1
\end{array}\right) e(\bar{Q})-\left(\begin{array}{c}
i+d-3 \\
d-2
\end{array}\right) e_{1}(\bar{Q}) \\
& =\left(\begin{array}{c}
i+d-2 \\
d-1
\end{array}\right) e(Q)-\left(\begin{array}{c}
i+d-3 \\
d-2
\end{array}\right) e_{1}(Q) .
\end{aligned}
$$


From the exact sequence

$$
0 \rightarrow Q^{i+1}: x / Q^{i} \rightarrow A / Q^{i} \stackrel{x}{\rightarrow} A / Q^{i+1} \rightarrow A /\left(Q^{i+1}, x\right) \rightarrow 0
$$

we get

$$
\ell\left(Q^{i} / Q^{i+1}\right)=\ell\left(A / Q^{i+1}\right)-\ell\left(A / Q^{i}\right) \leq \ell\left(A /\left(Q^{i+1}, x\right)\right)
$$

Using the above inequalities we obtain

$$
\begin{aligned}
\ell\left(A / Q^{n}\right) & =\sum_{i=1}^{n} \ell\left(Q^{i-1} / Q^{i}\right) \leq \sum_{i=1}^{n} \ell\left(A /\left(Q^{i}, x\right)\right) \\
& \leq \sum_{i=1}^{n}\left[\left(\begin{array}{c}
i+d-2 \\
d-1
\end{array}\right) e(Q)-\left(\begin{array}{c}
i+d-3 \\
d-2
\end{array}\right) e_{1}(Q)\right] \\
& =\left(\begin{array}{c}
n+d-1 \\
d
\end{array}\right) e(Q)-\left(\begin{array}{c}
n+d-2 \\
d-1
\end{array}\right) e_{1}(Q) .
\end{aligned}
$$

For every parameter ideal $Q$ of $A$, it is well known that $\ell(A / Q) \geq e(Q)$. The CohenMacaulay difference of $A$ with respect to $Q$ is defined by

$$
I(Q, A)=\ell(A / Q)-e(Q)
$$

Denote

$$
I(A)=\sup \{I(Q, A) \mid \text { for all parameter ideals } Q \text { of } A\} .
$$

By Corollary 2.4, if $\operatorname{dim}(A)=1$ and $Q$ is a parameter ideal of $A$ then

$$
e_{1}(Q)=-\ell\left(H_{\mathfrak{m}}^{0}(A)\right)
$$

This implies that if $A$ is an almost Cohen-Macaulay ring of dimension $d \geq 1$ and $Q=$ $\left(x_{1}, \ldots, x_{d}\right)$ is a parameter ideal of $A$ which $x_{1}, \ldots, x_{d-1}$ is a superficial sequence for $Q$, then

$$
e_{1}(Q)=e_{1}\left(\left(x_{d}\right), A /\left(x_{1}, \ldots, x_{d-1}\right)\right)=-\ell\left(H_{\mathfrak{m}}^{0}\left(A /\left(x_{1}, \ldots, x_{d-1}\right)\right)\right)
$$

is independent on $x_{d}$. This gives us the following bound.

Lemma 4.2. Let $A$ be a noetherian local ring of dimension $d \geq 2$ and $\operatorname{depth}(A) \geq d-1$. Let $x_{1}, \ldots, x_{d-1}$ be a subsystem of parameter of $A$ and $J=\left(x_{1}, \ldots, x_{d-1}\right)$. For all $n \geq 1$,

$$
I\left(A / J^{n}\right) \leq-\left(\begin{array}{c}
n+d-3 \\
d-2
\end{array}\right) e_{1}(Q) .
$$

Proof. We can add an element $x_{d}$ to the subsystem $x_{1}, \ldots, x_{d-1}$ such that $x_{1}, \ldots, x_{d-1}, x_{d}$ is a system of parameters of $A$ and let $B:=A /\left(x_{d}\right)$. Then

$$
\ell\left(A /\left(J^{n}, x_{d}\right)\right)=\ell\left(B / J^{n} B\right)
$$


By Lemma 4.1.

$$
\ell\left(B / J^{n} B\right) \leq\left(\begin{array}{c}
n+d-2 \\
d-1
\end{array}\right) e(J, B)-\left(\begin{array}{c}
n+d-3 \\
d-2
\end{array}\right) e_{1}(J, B) .
$$

Put $Q=\left(x_{1}, \ldots, x_{d}\right)$. We have $e(J, B)=e(Q, A)$. Without loss of generality we may assume that the residue field of $A$ is infinite and $x=x_{d}$ is superficial for $Q$. Since $A$ is almost Cohen-Macaulay, $e_{1}(Q B)=e_{1}(Q)$. Therefore,

$$
\ell\left(A /\left(J^{n}, x_{d}\right)\right) \leq\left(\begin{array}{c}
n+d-2 \\
d-1
\end{array}\right) e(Q)-\left(\begin{array}{c}
n+d-3 \\
d-1
\end{array}\right) e_{1}(Q) .
$$

By the associative formula for multiplicity,

$$
e\left(\left(x_{d}\right), A / J^{n}\right)=\sum_{\mathfrak{p}} \ell\left(A_{\mathfrak{p}} / J^{n} A_{\mathfrak{p}}\right) e\left(\left(x_{d}\right), A / \mathfrak{p}\right)
$$

where the sum is taken over all prime ideals $\mathfrak{p} \supseteq J$ with $\operatorname{dim}(A / \mathfrak{p})=1$. Since $A_{\mathfrak{p}}$ is a Cohen-Macaulay ring with $\operatorname{dim} A_{\mathfrak{p}}=d-1$ and $J A_{\mathfrak{p}}$ is a parameter ideal of $A_{\mathfrak{p}}$,

$$
\ell\left(A_{\mathfrak{p}} / J^{n} A_{\mathfrak{p}}\right)=\left(\begin{array}{c}
n+d-2 \\
d-1
\end{array}\right) \ell\left(A_{\mathfrak{p}} / J A_{\mathfrak{p}}\right)
$$

It follows that

$$
\begin{aligned}
e\left(\left(x_{d}\right), A / J^{n}\right) & =\left(\begin{array}{c}
n+d-2 \\
d-1
\end{array}\right) \sum_{\mathfrak{p}} \ell\left(A_{\mathfrak{p}} / J A_{\mathfrak{p}}\right) e\left(\left(x_{d}\right), A / \mathfrak{p}\right) \\
& =\left(\begin{array}{c}
n+d-2 \\
d-1
\end{array}\right) e\left(\left(x_{d}\right), A / J\right) \\
& =\left(\begin{array}{c}
n+d-2 \\
d-1
\end{array}\right) e(Q) .
\end{aligned}
$$

Hence

$$
I\left(\left(x_{d}\right), A / J^{n}\right)=\ell\left(A /\left(J^{n}, x_{d}\right)\right)-e\left(\left(x_{d}\right), A / J^{n}\right) \leq-\left(\begin{array}{c}
n+d-3 \\
d-2
\end{array}\right) e_{1}(Q) .
$$

Since $e_{1}(Q)$ is independent on $x_{d}$, this implies

$$
I\left(A / J^{n}\right)=\sup I\left(\left(x_{d}\right), A / J^{n}\right) \leq-\left(\begin{array}{c}
n+d-3 \\
d-2
\end{array}\right) e_{1}(Q) .
$$

Corollary 4.3. Let $A$ be a noetherian local ring of dimension $d \geq 2$ and $\operatorname{depth}(A) \geq d-1$. Let $x_{1}, \ldots, x_{d-1}$ be a subsystem of parameter of $A$ and $J=\left(x_{1}, \ldots, x_{d-1}\right)$. For all $n \geq 1$ and $t \geq 1$,

$$
\ell\left(J^{n}: x_{d}^{t} / J^{n}\right) \leq-\left(\begin{array}{c}
n+d-3 \\
d-2
\end{array}\right) e_{1}(Q)
$$


Proof. We have

$$
I\left(\left(x_{d}^{t}\right), A / J^{n}\right)=\ell\left(A /\left(J^{n}, x_{d}^{t}\right)\right)-e\left(\left(x_{d}^{t}\right), A / J^{n}\right) .
$$

By [3, Theorem 4.7.4] and [3, Theorem 4.7.6],

$$
e\left(\left(x_{d}^{t}\right), A / J^{n}\right)=\ell\left(A /\left(J^{n}, x_{d}^{t}\right)\right)-\ell\left(J^{n}: x_{d}^{t} / J^{n}\right) .
$$

Therefore

$$
\begin{aligned}
I\left(\left(x_{d}^{t}\right), A / J^{n}\right) & =\ell\left(A /\left(J^{n}, x_{d}^{t}\right)\right)-\left[\ell\left(A /\left(J^{n}, x_{d}^{t}\right)\right)-\ell\left(J^{n}: x_{d}^{t} / J^{n}\right)\right] \\
& =\ell\left(J^{n}: x_{d}^{t} / J^{n}\right) .
\end{aligned}
$$

Thus, by applying Lemma 4.2 we get

$$
\ell\left(J^{n}: x_{d}^{t} / J^{n}\right)=I\left(\left(x_{d}^{t}\right), J^{n}\right) \leq I\left(A / J^{n}\right) \leq-\left(\begin{array}{c}
n+d-3 \\
d-2
\end{array}\right) e_{1}(Q) .
$$

Lemma 4.4. Let $A$ be noetherian ring of dimension $d \geq 1$ and $\operatorname{depth}(A) \geq 1$. Let $I$ be an $\mathfrak{m}$-primary ideal of $A$ and $x$ be an superficial element for $I$. If $\operatorname{reg}(G(I /(x)) \leq m$, then there exists an integer $t$ such that

$$
\operatorname{reg}(G(I)) \leq m+\ell\left(I^{m+1}: x / I^{m}\right)+\ell\left(I^{m+t+1}: x^{t} / I^{m+1}\right) .
$$

Proof. Applying [10, Theorem 2.7] and [12, Lemma 2.2], we get the inequality in the lemma.

Theorem 4.5. Let $A$ be a noetherian local ring of dimension $d \geq 1$ and $\operatorname{depth}(A) \geq d-1$. Let $Q$ be a parameter ideal of $A$. Then

$$
\operatorname{reg}(G(Q)) \leq \begin{cases}\max \left\{-e_{1}(Q)-1,0\right\} & \text { if } d=1 \\ \max \left\{\left[-4 e_{1}(Q)\right]^{(d-1) !}+e_{1}(Q)-1,0\right\} & \text { if } d \geq 2\end{cases}
$$

Proof. Suppose that $Q$ is an ideal generated by system of parameter $x_{1}, \ldots, x_{d}$. Let $L=H_{\mathfrak{m}}^{0}(A)$. If $d=1$, then by 12 , Theorem 2.3] we have

$$
\operatorname{reg}(G(Q))=\max \{\ell(L)-1,0\}
$$

But in this case $e_{1}(Q)=-\ell(L)$ by Corollary 2.4. Hence

$$
\operatorname{reg}(G(Q))=\max \left\{-e_{1}(Q)-1,0\right\}
$$

If $d \geq 2$, we first consider that $A$ is a Cohen-Macaulay ring, then $\operatorname{reg}(G(Q))=0$. Therefore, we may assume that $A$ is not a Cohen-Macaulay ring. Then $\operatorname{depth}(A)=d-1$, so $e_{1}(Q) \leq-1$. Without loss of generality we may further assume that the residue field of 
$A$ is infinite. Then we may choose $x=x_{d} \in Q \backslash \mathfrak{m} Q$ such that $x$ is superficial for $Q$. Let $\bar{A}=A /(x)$ and $\bar{Q}=Q \bar{A}$. Since $A$ is not Cohen-Macaulay, $\bar{A}$ is not a Cohen-Macaulay ring. Because $A$ is an almost Cohen-Macaulay $\operatorname{ring}$ and $\operatorname{dim}(A)=d \geq 2, e_{1}(\bar{Q})=e_{1}(Q)$. Let $m=\operatorname{reg}(G(\bar{Q}))$. By Lemma 4.4 , there exists an integer $t$ such that

$$
\operatorname{reg}(G(Q)) \leq m+\ell\left(Q^{m+1}: x / Q^{m}\right)+\ell\left(Q^{m+t+1}: x^{t} / Q^{m+1}\right) .
$$

Set $J=\left(x_{1}, \ldots, x_{d-1}\right)$, we have

$$
\begin{aligned}
Q^{m+1}: x / Q^{m} & =\left(\left(x Q^{m}+J^{m} Q\right): x\right) / Q^{m} \\
& =\left(Q^{m}+\left(J^{m} Q: x\right)\right) / Q^{m} \\
& \cong\left(J^{m} Q: x\right) /\left(Q^{m} \cap\left(J^{m} Q: x\right)\right) .
\end{aligned}
$$

Since $J^{m} \subseteq Q^{m} \cap\left(J^{m} Q: x\right)$, we obtain

$$
\ell\left(Q^{m+1}: x / Q^{m}\right) \leq \ell\left(J^{m}: x / J^{m}\right) .
$$

On the other hand,

$$
\begin{aligned}
Q^{m+t+1}: x^{t} & =\left(x^{t} Q^{m+1}+J^{m+1} Q^{t}\right): x^{t}=Q^{m+1}+\left(J^{m+1} Q^{t}: x^{t}\right), \\
\ell\left(Q^{m+t+1}: x^{t} / Q^{m+1}\right) & =\ell\left(Q^{m+1}+\left(J^{m+1} Q^{t}: x^{t}\right) / Q^{m+1}\right) \\
& =\ell\left(\left(J^{m+1} Q^{t}: x^{t}\right) / Q^{m+1} \cap\left(J^{m+1} Q^{t}: x^{t}\right)\right) .
\end{aligned}
$$

Notice that

$$
\left(J^{m+1} Q^{t}: x^{t}\right) \subseteq\left(J^{m+1}: x^{t}\right) \quad \text { and } \quad J^{m+1} \subseteq Q^{m+1} \cap\left(J^{m+1} Q^{t}: x^{t}\right)
$$

This implies

$$
\ell\left(Q^{m+t+1}: x^{t} / Q^{m+1}\right) \leq \ell\left(J^{m+1}: x^{t} / J^{m+1}\right) .
$$

So,

$$
\begin{aligned}
\operatorname{reg}(G(Q)) & \leq m+\ell\left(Q^{m+1}: x / Q^{m}\right)+\ell\left(Q^{m+t+1}: x^{t} / Q^{m+1}\right) \\
& \leq m+\ell\left(J^{m}: x / J^{m}\right)+\ell\left(J^{m+1}: x^{t} / J^{m+1}\right) .
\end{aligned}
$$

Applying Corollary 4.3, we get

$$
\begin{aligned}
\operatorname{reg}(G(Q)) & \leq m-\left(\begin{array}{c}
m+d-2 \\
d-2
\end{array}\right) e_{1}(Q)-\left(\begin{array}{c}
m+d-1 \\
d-2
\end{array}\right) e_{1}(Q) \\
& \leq m-(m+1)^{d-2} e_{1}(Q)-(m+2)^{d-2} e_{1}(Q) .
\end{aligned}
$$

If $d=2$, we may put $m=-e_{1}(Q)-1$ and obtain

$$
\operatorname{reg}(G(Q)) \leq-3 e_{1}(Q)-1=\left(-4 e_{1}(Q)\right)^{(2-1) !}+e_{1}(Q)-1 .
$$


If $d>2$, by using induction on $d$ we may assume that

$$
m=\operatorname{reg}(G(\bar{Q})) \leq\left[-4 e_{1}(\bar{Q})\right]^{(d-2) !}+e_{1}(\bar{Q})-1 .
$$

From Lemma 2.5, we have $e_{1}(\bar{Q})=e_{1}(Q)$. Thus $m \leq\left(-4 e_{1}(Q)\right)^{(d-2) !}+e_{1}(Q)-1$. Because $m \geq-e_{1}(Q)$ and $-e_{1}(Q) \geq 1$, we obtain

$$
\begin{aligned}
\operatorname{reg}(G(Q)) & \leq m-(m+1)^{d-2} e_{1}(Q)-(m+2)^{d-2} e_{1}(Q) \\
& \leq-m e_{1}(Q)-(m+1)^{d-2} e_{1}(Q)-\sum_{i=0}^{d-2}\left(\begin{array}{c}
d-2 \\
i
\end{array}\right)(m+1)^{d-2-i} e_{1}(Q) \\
& \leq-\sum_{i=0}^{d-2}\left(\begin{array}{c}
d-1 \\
i+1
\end{array}\right)(m+1)^{d-2-i} e_{1}(Q) \\
& \leq-\sum_{i=1}^{d-1}\left(\begin{array}{c}
d-1 \\
i
\end{array}\right)(m+1)^{d-1-i} e_{1}(Q)^{i} \\
& \leq\left(m+1-e_{1}(Q)\right)^{d-1}+e_{1}(Q)-1 \\
& \leq\left[-4 e_{1}(Q)\right]^{(d-1) !}+e_{1}(Q)-1 .
\end{aligned}
$$

By Lemmas 2.1 and 2.2, $n(Q) \leq \operatorname{reg}(G(Q))$. From this we get the following corollary.

Corollary 4.6. Let $A$ be a noetherian local ring of dimension $d \geq 1$ and $\operatorname{depth}(A) \geq d-1$. Let $Q$ be a parameter ideal of $A$. Then

$$
n(Q) \leq \begin{cases}\max \left\{-e_{1}(Q)-1,0\right\} & \text { if } d=1 \\ \max \left\{\left[-4 e_{1}(Q)\right]^{(d-1) !}+e_{1}(Q)-1,0\right\} & \text { if } d \geq 2 .\end{cases}
$$

Let $R(Q)$ be the Rees algebra of $A$ with respect to $Q$. Ooishi [17, Lemma 4.8] proved that $\operatorname{reg}(G(Q))=\operatorname{reg}(R(Q))$. Represent $R(Q)=A[T] / J$, where $A[T]$ is a polynomial ring and $J$ is a homogeneous ideal of $A[T]$. The relation type $\operatorname{reltype}(Q)$ of $Q$ is defined as the largest degree of the minimal generators of $J$. It is well known 21, Corollary 1.3 and Proposition 4.1] that

$$
\operatorname{reltype}(Q) \leq \operatorname{reg}(R(Q))+1=\operatorname{reg}(G(Q))+1
$$

Therefore, we obtain the following bounds for the relation type of $Q$ in terms of $n(Q)$.

Corollary 4.7. Let $A$ be a noetherian local ring of dimension $d \geq 1$ and $\operatorname{depth}(A) \geq d-1$. Let $Q$ be a parameter ideal of $A$. Then

$$
\operatorname{reltype}(Q) \leq \begin{cases}\max \left\{-e_{1}(Q), 1\right\} & \text { if } d=1 \\ \max \left\{\left[-4 e_{1}(Q)\right]^{(d-1) !}+e_{1}(Q), 1\right\} & \text { if } d \geq 2\end{cases}
$$




\section{Acknowledgments}

The author would like to thank Hue University for partially support. The author is grateful to the referees for valuable comments and suggestions which help to create the final version.

\section{References}

[1] C. Blancafort, On Hilbert functions and cohomology, J. Algebra 192 (1997), no. 1, 439-459.

[2] M. Brodmann and C. H. Linh, Castelnuovo-Mumford regularity, postulation numbers and relation types, J. Algebra 419 (2014), 124-140.

[3] W. Bruns and J. Herzog, Cohen-Macaulay Rings, Cambridge Studies in Advanced Mathematics 39, Cambridge University Press, Cambridge, 1993.

[4] J. Elias, Depth of higher associated graded rings, J. London Math. Soc. (2) 70 (2004), no. $1,41-58$.

[5] _ On the last Hilbert-Samuel coefficient of isolated singularities, J. Algebra 394 (2013), 285-295.

[6] S. Goto and K. Ozeki, Uniform bounds for Hilbert coefficients of parameters, in: Commutative Algebra and its Connections to Geometry, 97-118, Contemp. Math. 555, Amer. Math. Soc., Providence, RI, 2011.

[7] L. T. Hoa, Reduction numbers and Rees algebras of powers of an ideal, Proc. Amer. Math. Soc. 119 (1993), no. 2, 415-422.

[8] _ Reduction numbers of equimultiple ideals, J. Pure Appl. Algebra 109 (1996), no. $2,111-126$.

[9] S. Huckaba and T. Marley, Hilbert coefficients and the depths of associated graded rings, J. London Math. Soc. (2) 56 (1997), no. 1, 64-76.

[10] C. H. Linh, Upper bound for the Castelnuovo-Mumford regularity of associated graded modules, Comm. Algebra 33 (2005), no. 6, 1817-1831.

[11] _ Castelnuovo-Mumford regularity and degree of nilpotency, Math. Proc. Cambridge Philos. Soc. 142 (2007), no. 3, 429-437.

[12] C. H. Linh and N. V. Trung, Uniform bounds in generalized Cohen-Macaulay rings, J. Algebra 304 (2006), no. 2, 1147-1159. 
[13] C. H. Linh and V. D. Trung, Hilbert coefficients and the depth of associated graded rings with respect to parameter ideals, to appear in Vietnam Journal of Mathematics.

[14] M. Mandal, B. Singh and J. K. Verma, On some conjectures about the Chern numbers of filtrations, J. Algebra 325 (2011), 147-162.

[15] T. Marley, The reduction number of an ideal and the local cohomology of the associated graded ring, Proc. Amer. Math. Soc. 117 (1993), no. 2, 335-341.

[16] L. Mccune, Hilbert coefficients of parameter ideals, J. Commut. Algebra 5 (2013), no. $3,399-412$.

[17] A. Ooishi, Genera and arithmetic genera of commutative rings, Hiroshima Math. J. 17 (1987), no. 1, 47-66.

[18] M. E. Rossi, N. V. Trung and G. Valla, Castelnuovo-Mumford regularity and extended degree, Trans. Amer. Math. Soc. 355 (2003), no. 5, 1773-1786.

[19] M. E. Rossi and G. Valla, Hilbert Functions of Filtered Modules, Lecture Notes of the Unione Matematica Italiana 9, Springer-Verlag, Berlin, 2010.

[20] A. Saikia and K. Saloni, Bounding Hilbert coefficients of parameter ideals, J. Algebra 501 (2018), 328-344.

[21] N. V. Trung, Reduction exponent and degree bound for the defining equations of graded rings, Proc. Amer. Math. Soc. 101 (1987), no. 2, 229-336.

[22] W. V. Vasconcelos, The Chern coefficients of local rings, Michigan Math. J. 57 (2008), $725-743$.

Cao Huy Linh

Department of Mathematics, College of Education, Hue University, 34 Le Loi, Hue, Vietnam

E-mail address: caohuylinh@@hueuni.edu.vn 